\title{
Was denken linguistische Laien über die (deutsche) Grammatik?
}

\section{Beobachtungen und Interpretationen anhand des öffentlichen Sprachgebrauchs}

Zusammenfassung: Im Beitrag wird zunächst die Frage nach dem Laien-Wissen über Grammatik in den größeren Kontext der Laienlinguistik bzw. folk linguistics gestellt. Ein besonderes Problem stellt in diesem Zusammenhang die Unterscheidung von Experten und Laien dar. Um diese Differenz besser in den Griff $\mathrm{zu}$ bekommen, wird ein graduierendes, merkmalorientiertes Vorgehen vorgeschlagen. Damit lässt sich ein realistischer Zugriff auf das komplexe Spannungsfeld zwischen Experten und Laien entwickeln. Im Weiteren werden auf einem korpuslinguistischen Fundament acht Basiskonzeptualisierungen identifiziert. Sie prägen das laienhafte Bild der Grammatik. Anhand dieser Basiskonzeptualisierungen kann man auch die Spannung zwischen dem Experten- und dem laienhaften Blick auf die Grammatik näher analysieren. Darin gibt es einige Übereinstimmungen, aber auch erhebliche Unterschiede.

Abstract: In the article the question of lay knowledge of grammar is first of all put into the larger context of folk linguistics and ,Laienlinguistik‘. A particular problem in this context is the distinction between experts and laypeople. In order to get a better grip on this difference, a gradual, feature-oriented approach is proposed. This allows a realistic access to the complex field between experts and laypeople to be developed. Furthermore, eight basic conceptualizations (,Basiskonzeptualisierungen') are identified on a corpus-linguistic foundation. They shape the lay image of grammar. On the basis of these Basiskonzeptualisierungen it is also possible to analyze the tension between the expert and the layman's view of grammar in more detail. There are some similarities, but also considerable differences.

Schlüsselwörter: Laienlinguistik, folk linguistics, Sprachbewusstsein, language awareness, Spracheinstellung, language attitude, Sprachnorm, linguistic norm, Deutsch, German

Klein, Wolf Peter: Institut für Deutsche Philologie, Universität Würzburg, Am Hubland, D-97074 Würzburg, wolfpeter.klein@uni-wuerzburg.de 


\section{Einführung}

Laienlinguistik und folk linguistics können als moderne Bindestrichlinguistiken verstanden werden, obwohl die beiden Wörter gar keine Bindestriche enthalten. Angesichts der langen Geschichte der Sprachwissenschaft verkörpern sich hinter den beiden Begriffen jedenfalls relativ neue Arbeitsbereiche. Dafür stehen beispielsweise die einführenden Werke von Antos (1996) und Niedzielski \& Preston (2003) (ähnlich Wilton \& Stegu 2011), in denen das Feld richtungweisend präsentiert wurde. Ferner existieren Spezialstudien, in denen etwa bestimmte sprachbezogene Publikationsformen (z. B. Strauss 2018) oder lexikalische Selektionsprozesse (Meciarova 2015) näher betrachtet wurden. Zum Kontext gehört auch die jüngste Konjunktur der sog. Wahrnehmungsdialektologie (perceptual dialectology, vgl. Anders, Hundt \& Lasch 2010; Hundt 2018).

Die wissenschaftlichen Ansatzpunkte von Laienlinguistik und folk linguistics sind nicht völlig identisch, überschneiden sich aber sicher an vielen Punkten. In beiden Fällen steht im Kern die Überzeugung, dass es sprachwissenschaftlich ergiebig und weiterführend sein kann, ausdrücklich das sprachbezogene Wissen der Nutzer einer Sprache zu erforschen und systematisch in die linguistische Programmatik zu integrieren. Die besondere Stoßrichtung dieser Forschungsrichtungen liegt dabei in der - zumindest impliziten - Gegenüberstellung von linguistischem und nicht-linguistischem Wissen. Demnach gibt es einen mehr oder weniger großen Unterschied zwischen dem, was Linguisten über Sprachen wissen und herausgefunden haben, und dem, was im Kopf von Nicht-Linguisten (Laien, folk) vorhanden ist. Die Brisanz dieser Differenz scheint geradezu der Motor gewesen zu sein, warum diese Arbeitsbereiche entstanden sind und mit bestimmten sprachlichen Etiketten versehen wurden. Linguisten denken, so die Unterstellung, anders über Sprache(n) nach als Nicht-Linguisten. Die grundsätzlichen Fragen wären dann: Wie genau ist das Wissen des linguistischen Laien über Sprache beschaffen und entstanden? Wie unterscheidet es sich vom Wissen des Sprachwissenschaftlers? Und weiter gedacht: Welche Auswirkungen könnten bestimmte laienlinguistische Wissenselemente auf die Entwicklung der einzelnen Sprachen und die sprachliche Kommunikationsformen insgesamt gehabt haben und gegenwärtig immer noch haben?

Dabei sind die Ambitionen von Laienlingustik und folk linguistics, etwas allgemeiner betrachtet, keineswegs völlig neu oder gar revolutionär, gleichzeitig vielfach anschlussfähig $\mathrm{zu}$ anderen, teilweise bereits seit langem etablierte Forschungsfeldern der Sprachwissenschaft. In den verschiedensten Formen beschäftigte man sich in der vergangenen und der aktuellen Sprachwissen- 
schaft mit dem, was mit den Begriffen Laienlinguistik und folk linguistics programmatisch in den Vordergrund gerückt wird. Ich erinnere nur an solche disziplinären Etiketten wie Sprachbewusstseinsforschung bzw. Sprachbewusstseinsgeschichte, Spracheinstellungsforschung, Volksetymologie und Sprachmythologie. Um nur einige Impressionen zur existierenden Literatur und den damit verbundenen Forschungsansätzen und Forschungsergebnissen ins Spiel zu bringen: Dem Begriff der Volksetymologie können seit dem 19. Jahrhundert (wegweisend Andresen 1876) verschiedene Arbeiten zugeschrieben werden (resümierend Olschansky 1996). Sprachmythologisch wurde aus historischer Perspektive vor allem das zentrale christliche Theorem des Turmbaus von Babel beleuchtet (Borst 1957-1963). Was man im Alltag über (die deutsche) Sprache denkt, ist das zentrale Thema der Spracheinstellungsforschung. Sie arbeitet häufig mit statistischen Daten (z. B. Adler \& Plewnia 2019; Deutsche Akademie für Sprache und Dichtung 2013, 2017; Eichinger et al. 2009; Gärtig, Plewnia \& Rothe 2010) und kann ganz unterschiedliche Angriffspunkte besitzen, z. B. anschließend an orthographische Variation (Klotzsche \& Reich 2016), an die Textebene (Peter 2011), an diatopische Varietäten (Cuonz 2014; Hundt 1992; Plewnia \& Rothe 2012; Regener 2000) oder mit historischer Blickrichtung (Ziegler 1999). Auch Arbeiten in denen „populäre Irrtümer über Sprache“ (Ernst, Freienstein \& Schaipp 2011) oder „populärwissenschaftliches Schreiben über sprachliche Fragen“ (Niederhauser 1997) thematisiert werden, gehen in eine ähnliche Richtung, allerdings mit einem anderen konzeptionellen Schwerpunkt. Was schließlich den Begriff Sprachbewusstsein angeht, reichen die Arbeiten einerseits tief in die Sprach- und Wissenschaftsgeschichte hinein (z. B. Huber 1984; Klein 1992; Leweling 2005; Scharloth 2005), letztlich sogar zurückgehend bis zur Antike (Fögen 2000; Müller 2001). Andererseits stehen hier didaktische und (schrift-)spracherwerbsorientierte Perspektiven im Raum (z. B. Andresen 1985), zudem sprachpolitische (z. B. Glück 1979) und sprachtheoretische (z. B. Schmidlin, Behrens \& Bickel 2015) Thematisierungen.

Bei fast allen diesen Forschungen hat sich herausgestellt, dass das Sprachwissen der Laien und die alltägliche Sprachreflexion sehr häufig auf Wertungen zielt. Auf- und Abwertungen spielen in der kommunikativen Realität offensichtlich eine sehr prominente Rolle. Neutralität, Nüchternheit und Objektivität sind keine Haltungen, die man in sprachlichen Dingen bevorzugt. Richtig vs. falsch, gut vs. schlecht, schön vs. hässlich (und viele andere mehr!) sind typische Wertadjektive, mit denen solche sprachbezogenen Beurteilungen vollzogen werden. Mit derartigen Wort-Etiketten können faktisch alle sprachlichen Einheiten belegt werden. Um nur einige aufzuzählen: Die Wertungen mögen sich auf einzelne Wörter, Wortbildungen oder Wortschatzbereiche, aber auch auf einzelne 
Flexionsformen oder syntaktische Konstruktionen isolierter Sätze beziehen. Ebenso ist es möglich, dass bestimmte Aussprachen oder sogar spezifische Einzellaute als gut oder schlecht usw. charakterisiert werden, genauso wie ganze Varietäten, oft die diatopisch verankerten, oder aber einzelne Texte bzw. Textmuster. In sprachlichen Dingen ist vor der Bewertungsfreudigkeit der Laien faktisch nichts sicher. Vielleicht könnte man das sogar noch verschärfen. Statt Bewertungsfreudigkeit findet man auf diesem Feld oft sogar eine Bewertungssucht, gelegentlich sogar einen Bewertungsrausch bis hin zu regelrechten evaluativen Empörungen, oft mit starker sozialdistinktiver Funktionalität. Demnach sagt die jeweils bewertete Sprache auch immer etwas über die jeweiligen Sprecher aus. Als Beispiel mögen dazu zwei derzeit relativ verbreitete laienlinguistische Bewertungswörter für graphematische Variation dienen: DeppenApostroph und Deppen-Leerzeichen. Mit anderen Worten, wertende Urteile werden im Laiendiskurs über Sprache schnell und lustvoll gefällt, objektives und nüchternes Faktenwissen ist dagegen weniger verbreitet.

Vor diesem Hintergrund verwundert es nicht, dass die Differenz zwischen dem Sprachwissen der Laien und den Erkenntnissen und Haltungen der Sprachwissenschaft, milde gesagt, von konstitutiven Konflikten durchzogen ist. Es scheint so zu sein, dass hier grundsätzlich unterschiedliche Sichtweisen herrschen. Denn in der Sprachwissenschaft hält man sich mit Wertungen zurück. Man strebt schließlich, wie in jeder Wissenschaft, danach, die Objekte seines Tuns neutral, nüchtern und objektiv wahrzunehmen und $\mathrm{zu}$ untersuchen. Normalerweise findet man in der Sprachwissenschaft keine Rede davon, dass ein sprachlicher Befund richtig oder falsch, gut oder schlecht, schön oder hässlich ist oder auch nur sein könnte. Eine (terminologische) Facette dieser Spannung liegt darin, dass sich die Sprachwissenschaft zu einer deskriptivanalytischen Haltung bekennt, während das Sprachwissen der Laien auf einer präskriptiv-normativen Basis ruhen soll (relativierend Klein 2004).

Vor diesem Hintergrund möchte ich im vorliegenden Text an einem Detail des Laienwissens über Sprache einsetzen. Es soll darum gehen, welches Bild die Laien von einem zentralen Bereich der Sprache, nämlich der Grammatik, besitzen. Dabei will ich mich ausschließlich auf den Geltungsbereich der deutschen Sprache und Grammatik beziehen. Das schließt freilich gelegentliche Ausblicke auf nicht-deutsche Verhältnisse nicht aus.

Zur Frage, wie linguistische Laien die Grammatik sehen und was sie darüber wissen, existieren bisher keine ausgebauten, belastbaren Studien. Zwar gibt es in der linguistischen Literatur immer wieder Bemerkungen dazu, dass die Grammatik für Laien mit bestimmten Konnotationen und Einschätzungen verbunden, besser vielleicht: befrachtet ist (z. B. Niedzielski \& Preston 2003: 
Kap. 4.2.2). So tauchen in (angelsächsischen) Laien-Kontexten bei explizit formulierten Perspektiven auf Grammatik etwa häufig Wörter und Wendungen wie torture, good grammar, correct, proper/improper, uppity u. ä. auf (Niedzielski \& Preston 2003: 229-231, 234). Wie diese Befunde aber im Detail zusammenhängen und ob sie über bloße Impressionen und Einzelmeinungen hinausgehen, wurde bisher noch nicht genauer ausgelotet.

Dabei ist zumindest die Hypothese im Hinterkopf $\mathrm{zu}$ behalten, dass Laienwissen und Expertenwissen über Sprache sich nicht nur beim Status von Bewertungen unterscheiden. Denn hinter der Differenz von Laien- und Expertenwissen könnten auch unterschiedliche kognitive Strukturen stehen. Laienwissen scheint nämlich, so die plausibel untermauerte These von Hennig (2016: 10), eher essentialistisch-kategorial, Expertenwissen eher relational-konzeptuell determiniert zu sein. Etwas zugespitzt und vergröbert kann man demnach davon ausgehen, dass Laienwissen über Grammatik vor allem an Einzelheiten (z. B. einzelnen Wörtern) ansetzt, während Expertenwissen eher an abstrakteren Strukturen und (morphologischen, lexikalischen, syntaktischen) Relationen orientiert ist. Auch wenn man den Begriff der Subjektivität in den Mittelpunkt stellt, kommt man $\mathrm{zu}$ interessanten Überlegungen, wie sich das grammatische Wissen von Laien und Experten nicht nur inhaltlich, sondern strukturell-formal unterscheiden könnte (vgl. Hoffmeister 2020).

Bevor allerdings die Frage nach dem Laienwissen über Grammatik in $\mathrm{Au}$ genschein genommen werden soll, möchte ich zuvor erst einmal den Begriff des linguistischen Laien näher betrachten und problematisieren (Abschnitt 2). Denn wer etwas über das Laienwissen zur Grammatik sagen möchte, muss zumindest andeuten, wie er den Begriff des Laien versteht und näher definiert. Danach werden in einem kürzeren Abschnitt die methodologischen Leitlinien erläutert (Abschnitt 3), auf deren Basis dann bestimmte Erkenntnisse zur Laiensicht auf die Grammatik formuliert werden (Abschnitt 4).

\section{Was sind linguistische Laien?}

Die Unterscheidung zwischen linguistischen Laien und Experten wird in einschlägigen Untersuchungen oft als bekannt und mehr oder weniger unproblematisch vorausgesetzt (z. B. Anders 2010; Berthele 2010, weiterführend dagegen Beuge 2019: Kap. 2; Hoffmeister 2019). Erst einmal ist dieses Vorgehen natürlich plausibel, weil man sich auf der Basis der beiden Begriffe durchaus sinnvoll verständigen kann. Schließlich sind deren Bedeutungen bekannt und einigermaßen fest umrissen. Sie sichern normalerweise eine unproblematische Kom- 
munikation. Im Fall von Experte kommen dazu noch Wörter wie Fachmann/Fachfrau und vielleicht Profi, Sachkundiger, Spezialist. Direkte Gegenstücke zum neutralen Laie sind dagegen schwieriger zu finden, weil hier von vorneherein nur eher abwertende Wörter im Raum stehen (z. B. Anfänger, Dilettant, Ignorant). Angesichts dieses Sachstands kommt dem Plädoyer für die Nutzung des Worts Amateur (vgl. Hoffmeister 2019) eine große Plausibilität zu. Trotz diesem Ungleichgewicht kann man bei diesen Wörtern davon ausgehen, dass man normalerweise einen linguistischen Laien trennscharf und unzweifelhaft von einem Experten unterscheiden können sollte.

Die Differenz zwischen diesen Personenkreisen lässt sich im ersten Schritt negativ bestimmen. Ein linguistischer Laie (auch: Laien-Linguist) ist eine Person, die sich mit Sprachwissenschaft nicht auskennt, Sprachwissenschaft nicht studiert hat und insofern keine linguistischen Kenntnisse besitzt. Eine solche Bestimmung eröffnet dann auch die Möglichkeit, Laienwissen und Expertenwissen zu kontrastieren. Auch dies erfolgt oft in negativen Kategorien, insofern das Laienwissen als ein Wissen gesehen wird, das bestimmte Eigenschaften, die für Expertenwissen konstitutiv sein sollen, eben nicht besitzt. So wurde das Laienwissen beispielsweise als „implizit, lückenhaft, wenig elaboriert und selten durchstrukturiert [...] mehrdeutig, formal inkohärent und inhaltlich inkonsistent [...] falsifikationsresistent und damit immunisierungsanfällig“ (Antos 1996: 32-33) charakterisiert, zudem mit Hinweis darauf, dass die Unterschiede zum Expertenwissen manchmal „nur graduell“ (Antos 1996: 34) sein mögen.

Vor diesem Hintergrund kann man sich einen Überblick darüber verschaffen, welchen Status der Laien-Begriff in empirischen Untersuchungen tatsächlich besitzt. Wer wird im Rahmen einschlägiger Erhebungen also als linguistischer Laie hinzugezogen, um mehr über Laien-Linguistik zu erfahren? Im einzelnen gilt etwa, dass Studierende der Germanistik im 1./2. Semester als Laien verstanden wurden (vgl. Hundt 2010: 185). Auf dieser Linie schloss man (fertig ausgebildete!?) Deutschlehrer von einer Untersuchung linguistischer Laien aus (vgl. Beuge 2014: 141). In ähnlicher Art und Weise wurden „Studentinnen und Studenten mit keinen bzw. nur sehr geringen linguistischen Vorkenntnissen“ für eine Datenerhebung herangezogen (Spiekermann 2010: 226227). „Studierende, MitarbeiterInnen des Schweizer Fernsehens DRS, die am Bildschirm als ModeratorInnen auftreten“, fungierten in einer anderen Untersuchung als linguistische Laien (Christen 2010: 272). Meciarova (2015: 14) dagegen nahm schlicht die Einsendungen zu den „öffentlichen Wortwahlen“ (Das schönste deutsche Wort, Unwort des Jahres) als Belege für „laienhafte“ Sprachreflexion, ohne genaueres über die Autoren dieser Einsendungen zu wissen. In einer weiteren Untersuchung spielten rezeptionsorientierte Begriffe eine Rolle 
für die Identifikation von Laien-Linguisten. Weil bestimmte Rhetorik-Ratgeber sich an ein „fachexternes Publikum“ (Strauss 2018: 141) richten, wurden sie als Instanzen von Laien-Linguistik gesehen. Das involvierte sogar akademisch gebildete Autoren wie Psychologen, Soziologen, Betriebswirtschaftler und selbst Kommunikationswissenschaftler - nach der Autorin dieser Studie also alles Laien-Linguisten (vgl. Strauß 2018: 141).

Diese Impressionen zum Umgang mit dem Begriff des linguistischen Laien zeigen, dass Untersuchungen zur Laien-Linguistik von heterogenen Ansatzpunkten ausgehen können. Mit einer binären Gegenüberstellung von linguistischen Laien und Experten ist es insofern nicht so einfach, wie anfangs vielleicht gedacht. Stattdessen muss davon ausgegangen werden, dass es zwischen bestimmten (prototypischen) Experten und Laien ein gewisses Übergangsfeld gibt. Eine realistische Theorie der Laien-Linguistik hat also mit graduellen Unterschieden zu rechnen, die über die einfache Dichotomie von linguistischen Laien und Experten hinausgehen. Mit den Worten von Davies (2010: 386):

Am einen Pol finden wir professionelle SprachwissenschaftlerInnen und am anderen stehen die Menschen, die überhaupt keine sprachwissenschaftlichen Kenntnisse besitzen. Dazwischen befinden sich Menschen mit sehr unterschiedlich ausgebauten metasprachlichen Kompetenzen.

Die Erkenntnis von der Gradualität des Verhältnisses von linguistischen Laien und Experten wurde in der Forschung also schon formuliert. Sie scheint allerdings (noch) nicht zum sprachwissenschaftlichen Gemeingut zu gehören. Methodologisch und terminologisch bleibt sie oft etwas unterbelichtet, wurde jedenfalls nicht systematisch weiterverfolgt.

Die Perspektiven zur Erforschung laienlinguistischen Wissens lassen sich an dieser Stelle also schärfen: Die simple Dichotomie zwischen LaienLinguisten und professionellen Linguisten ist für weitergehende Analysen nicht wirklich tragfähig. Stattdessen muss dieses Verhältnis graduell, nicht dichotom verstanden werden. Welche Aspekte, zumindest potentiell, herangezogen werden können, um diese Differenzierung je nach Untersuchungskontext zu leisten, kann exemplarisch der folgenden Tabelle entnommen werden. Darin werden je nach Zeile unterschiedliche Stufen zwischen (prototypischen) LaienLinguisten und professionellen Linguisten durch Anwesenheit (x) bestimmter Eigenschaften gekennzeichnet. Die hier angeführten Eigenschaften könnten zweifellos noch durch weitere Aspekte ergänzt und damit das jeweilige Untersuchungsprofil für bestimmte Zwecke geschärft werden: 
Tab. 1: Laien und Experten im graduierenden Kontrast.

\begin{tabular}{|c|c|c|c|c|c|c|c|c|}
\hline & \multicolumn{3}{|c|}{ Ausbildung } & \multirow{2}{*}{$\begin{array}{l}\text { Interesse } \\
\text {... für die } \\
\text { (deutsche) } \\
\text { Sprache }\end{array}$} & \multirow{2}{*}{$\begin{array}{l}\text { Beruf } \\
\text { arbeitet } \\
\text { als Lin- } \\
\text { guist }\end{array}$} & \multirow[b]{2}{*}{$\begin{array}{l}\text { arbeitet } \\
\text { als } \\
\text { Sprach- } \\
\text { lehrer }\end{array}$} & \multirow[b]{2}{*}{$\begin{array}{l}\text { arbeitet in } \\
\text { sprachin- } \\
\text { tensivem } \\
\text { Beruf }\end{array}$} & \multirow[b]{2}{*}{$\stackrel{0}{\stackrel{0}{N}}$} \\
\hline & $\begin{array}{l}\text { Studi- } \\
\text { um der } \\
\text { Lingu- } \\
\text { istik }\end{array}$ & $\begin{array}{l}\text { z. T. } \\
\text { Linguis- } \\
\text { tik- } \\
\text { Studium }\end{array}$ & $\begin{array}{l}\text { Studium, } \\
\text { aber kei- } \\
\text { ne Lingu- } \\
\text { istik }\end{array}$ & & & & & \\
\hline \multirow[t]{9}{*}{ Laie } & & & & & & & & 1 \\
\hline & & & & $x$ & & & & 2 \\
\hline & & & $x$ & $x$ & & & & 3 \\
\hline & & & $\mathrm{x}$ & $x$ & & & $x$ & 4 \\
\hline & & & $x$ & & & & $\mathrm{x}$ & 5 \\
\hline & & $x$ & & $x$ & & & $x$ & 6 \\
\hline & & $x$ & & $x$ & $x$ & & & 7 \\
\hline & $x$ & & & $x$ & & & $x$ & 8 \\
\hline & $x$ & & & $x$ & & $x$ & & 9 \\
\hline Experte & $x$ & & & $x$ & $x$ & & & 10 \\
\hline
\end{tabular}

Je nachdem, welche Ausbildung eine Person genossen hat, welche Interessen sie pflegt und welchen Beruf sie momentan ausübt, entstehen unterschiedliche Gruppierungen. Ein prototypischer Laie wäre dann eine Person, die nicht studiert hat, kein Interesse für Sprache hegt und auch beruflich direkt nichts mit Sprache zu tun hat (= Zeile 1). Demgegenüber stände ein studierter Sprachwissenschaftler, der als solcher beruflich tätig ist (= Zeile 10). Dazwischen sind nun verschiedene Konstellationen denkbar, in denen Experten- und Laien-Status ein Stück weit verschwimmen können. Man denke beispielsweise an einen Journalisten, der womöglich Politologie studiert hat. In seinem beruflichen Alltag hat er sehr viel mit Sprache zu tun, daran womöglich auch ein besonderes Interesse, das aber aufgrund seiner Ausbildung nicht in ein Studium der Sprachwissenschaft eingebettet ist (= Zeile 4). Oder man stelle sich einen Historiker vor, der als Lehrer arbeitet, also ebenfalls in einem sprachintensiven Beruf tätig ist. In seinem Studium hat er vielleicht das eine oder andere sprachwissenschaftliche Seminar besucht (= Zeile 6). Ist das nun ein linguistischer Laie oder ein linguistischer Experte? Hier hilft nur eine begründete Fein-Differenzierung weiter, weil alles andere die Komplexität der gegebenen Tatsachen nicht adäquat wiedergeben würde. 
Die Charakterisierungen aus Tab. 1 sind nur als Beispiele zu verstehen. Untersuchungen darüber, was Laien über die Grammatik denken, wären demnach mit einem genaueren Bezug auf bestimmte Personengruppen zu korrelieren. Wie das im Einzelnen erfolgt, ist vom übergeordneten Erkenntnisinteresse und dem jeweiligen Projektrahmen abhängig zu machen. Wer - etwa in Fragebogenuntersuchungen - mehr über das Wissen von linguistischen Laien herausfinden möchte, müsste zum Beispiel persönliche Metadaten erheben, die Ausbildung, (aktuellen) Beruf und Interesse an Sprache betreffen. Die simple Kontrastierung von linguistischen Laien und Experten steht immer in der Gefahr, ein verzerrtes Bild der - durchaus besser greifbaren - Wirklichkeit zu zeichnen. Das heißt natürlich nicht, dass die einfache Rede von linguistischen Laien völlig haltlos wäre.

\section{Zur Methodologie: Kollokationen und Basiskonzeptualisierungen}

Die Überlegungen des vorangegangenen Kapitels haben auf ein methodologisches Problem bei den Untersuchungen zur Laien-Linguistik hingewiesen. An der Problematisierung der Frage, was eigentlich ein Laien-Linguist ist, wurde mittelbar auch schon deutlich, dass es zu einer Erforschung der LaienLinguistik unterschiedliche Zugänge geben kann. Daran sei angeknüpft, wenn ich nun einen Ansatz skizzieren möchte, mit dem sich eine kleine Tiefenbohrung zu der eingangs genannten Fragestellung - wie sehen linguistische Laien die Grammatik? - anstellen lässt. Dabei wurde die Klärung des Problems, inwiefern man bei empirischen Erhebungen systematisch zwischen linguistischen Laien und Experten unterscheiden kann, freilich bewusst umgangen. Methodisch möchte ich nämlich am öffentlichen Sprachgebrauch anknüpfen, wie er in den großen Korpora des Deutschen vorhanden und analysierbar ist (DeReKo, DWDS/Deutsches Textarchiv (DTA)). Dabei werde ich vor allem Kollokationen aufgreifen und gehe insofern von folgendem Untersuchungsszenario aus:

Ich nehme zunächst an, dass die öffentliche Sprache, die in den o. g. Korpora präsent ist, für einen nicht-fach(sprach)lichen Diskurs steht. Diese Annahme ist wohl unmittelbar gerechtfertigt, da hier in erster Linie Presse-Texte, literarische Werke sowie Alltagstexte enthalten sind. Es handelt sich also nicht um ein sprachwissenschaftliches Fachtextkorpus. Gegen diese Annahme soll hier auch nicht sprechen, dass bei den Autoren dieser Texte sicher auch die eine oder andere Person dabei sein wird, die (deutsche) Sprachwissenschaft studiert hat. 
Vor allem im DTA findet man sogar einige (historische) Texte von Sprachwissenschaftlern, z. B. von Jacob Grimm, Wilhelm von Humboldt, Daniel Sanders. Diese Anwesenheit linguistischer Fachsprache und Kompetenz ist aber angesichts des Umfangs der großen Korpora sehr begrenzt und statistisch faktisch zu vernachlässigen. Sie führt nicht dazu, dass diese Korpora ihren Laien-Status verlieren und einen fachwissenschaftlichen Charakter bekommen. Die Textsammlungen bleiben als Ganze deutlich nicht-fachlich geprägt und können insofern einen Zugang zu dem verschaffen, was linguistische Laien über Sprache (hier: Grammatik) denken.

Als Spiegelungen des laienlinguistischen Wissens möchte ich statistisch signifikante Kollokationen des öffentlichen Sprachgebrauchs heranziehen, namentlich diejenigen, in denen die Wörter (bzw. Phrasen) (deutsche) Grammatik und grammatisch/grammatikalisch eine tragende Rolle spielen. Einfach gesagt, zeigen mir solche Kollokationen an, in welchen inhaltlichen Perspektiven und thematischen Zusammenhängen nicht-fachlich über Grammatik gesprochen wird. Diese Präsenz baut, so die Voraussetzung, auf einem Wissen auf, das in den genannten Kollokationen greifbar wird, dort diskursstrukturell Gestalt annimmt und gegebenenfalls auch kognitiv prägende Wirkungen entfaltet. Die typischen, korpuslinguistisch konkret bezifferbaren Muster des Sprachgebrauchs geben demnach Hinweise darauf, wie linguistische Laien die (deutsche) Grammatik konzeptualisieren und von Fall zu Fall ihr Wissen in die öffentliche Sprache einfließen lassen. Typische Sprachmuster erlauben Rückschlüsse auf gängiges Alltagswissen. Statistisch signifikante Kollokationen stehen für mehr als Sprache, nämlich für kommunikativ verkörperte Meinungen und Wissensbestände, die qua musterhaften Sprachformen in größeren kommunikativen Umgebungen verbreitet und stabilisiert werden.

Um es an einem einfachen Beispiel zu exemplifizieren: Wenn das Wort Grammatik im Korpus überdurchschnittlich häufig mit dem attributiven Adjektiv korrekt vorkommt, so ist das ein Zeichen dafür, dass die Grammatik im Sprachwissen deutlich mit Korrektheits- bzw. Inkorrektheitsurteilen verbunden ist. Kognitiv gewendet: Wem Grammatik auf der Zunge liegt, bei dem sind Wörter wie korrekt/inkorrekt nicht fern. Er sieht die Grammatik also typischerweise, zumindest assoziativ, durch die Brille von Korrektheitserwägungen. Der Umstand, dass Grammatik oft in syntaktischen Koordinationen mit den Substantiven Rechtschreibung, Syntax und Wortschatz verbunden ist, indiziert ferner, dass man sie in einer Reihe mit anderen Bestandteilen einer Sprache begreift. Diesen Zusammenhang kann man wiederum als einen Hinweis auf das Alltagsbewusstsein vom vielschichtigen Aufbau der Sprache interpretieren. 
Um solche Zusammenhänge systematisch zu ermitteln, wurden also verschiedene Strukturprofile von Kollokationen aus den o. g. Korpora abgefragt bzw. ermittelt. An erster Stelle seien die Kollokationen zu Grammatik und grammatisch/grammatikalisch aus den sog. „Wortprofilen“ des DWDS genannt (ermittelt 05.08.2019). Syntaktisch kommen damit die folgenden (typischen) Sprachmuster ins Spiel:

- Grammatik hat Adjektivattribut x,

- Grammatik hat Genitiv-Attribut x,

- Grammatik steht in syntaktischer Koordination mit x,

- Grammatik ist in einer Präpositionalphrase enthalten,

- Grammatik ist Akkusativ- oder Dativ-Objekt vom Verb x,

- grammatisch/grammatikalisch ist Adjektivattribut zum Substantiv x,

- grammatisch/grammatikalisch ist Adverbialbestimmung zu x

In die Auswertung der DWDS-Daten flossen jeweils die zehn oberen Ränge in den Kollokationstabellen solcher Wortprofile ein.

Die einschlägigen Kookkurrenzen zu denselben Wörtern wurden im DeReKo über Cosmas II erhoben (ermittelt 26.04.2019/06.05.2019). Beim DeReko ergaben die Kookkurrenzanalysen die folgenden Trefferlisten: Grammatik (25.638 Treffer), grammatikalisch (3.962 Treffer), grammatisch (1.970 Treffer). In die Auswertung gingen hier jeweils die ersten 100 Treffer dieser Trefferlisten ein. Da die Trefferlisten nach statistischer Signifikanz der Beiwörter (log-likelihoodratio (LLR)) geordnet sind, kann man davon ausgehen, dass man die primären Beiwörter in den Händen hat, wenn man sich auf die oberen 100 Wörter der Trefferlisten konzentriert (zu den korpuslinguistisch-methodologischen Hintergründen genauer Perkuhn \& Belica 2004). Sie sind statistisch besonders einschlägig mit Grammatik und grammatisch/grammatikalisch verbunden. Außerdem wurde im DeReKo eine Kookkurrenzanalyse zur Phrase deutsche Grammatik durchgeführt (11.03.2019). Sie lieferte 800 Belege, die im Einzelnen auf typische Verwendungsweisen überprüft wurden. Daraus ergab sich, dass die genannte Phrase 88-mal als Subjekt in einem Aktiv-Satz erschien, 30-mal als Subjekt in einem Passiv-Satz und 285-mal als Akkusativ-Objekt.

Die im folgenden Kapitel zu erläuternden Ergebnisse betreffen also in allen Fällen zunächst signifikante Sprachgebrauchsmuster zu den Wörtern (deutsche) Grammatik und grammatisch/grammatikalisch. Das ist sozusagen das korpuslinguistische Fundament für alles weitere. Aus den statistisch besonders prominent profilierten Kollokationen wurden dann in einem zweiten Schritt sog. Basiskonzeptualisierungen zur Grammatik erschlossen (im Folgenden abgekürzt als BK). Bestimmte kollokative Muster weisen nämlich thematisch oft in diesel- 
be Richtung. Zum obigen Beispiel korrekt/inkorrekt passen etwa häufige Kollokationen mit den Wörtern richtig/falsch. Zusammen mit anderen typischen Beiwörtern ergibt sich eine Basiskonzeptualisierung, die man wie folgt formulieren kann: „Grammatik kann korrekt (richtig) bzw. inkorrekt/unkorrekt (falsch) sein." (siehe unten = BK 5).

Solche Basiskonzeptualisierungen sind insofern interpretative Resultate von Abstraktions- bzw. Additionsprozessen, die auf korpuslinguistischer Grundlage erfolgen. Empirisch basieren sie zwar auf statistisch signifikanten Kollokationen. Sie (und die Art ihrer Formulierung) sind als solche allerdings nicht direkt in den Kollokationsdaten verankert, sondern müssen daraus per abstrahierender Auslegung herausgefiltert werden. Das geschieht dadurch, dass man in den Ergebnislisten semantisch ähnliche Kollokationsbefunde identifiziert und, wie oben exemplifiziert, zu Basiskonzeptualisierungen zusammenfasst. Es lässt sich dann ferner annehmen, dass die Basiskonzeptualisierungen, die sich so aus dem Sprachgebrauch großer Korpora abstrahieren lassen, Hinweise auf das Wissen und die Einstellungen zur Grammatik von LaienLinguisten geben. Was im Diskurs immer wieder in denselben oder in ähnlichen Sprachformen und -mustern auftaucht, ist ein Zeichen für eine bestimmte stabile Formation im kognitiven Leben der Diskursteilnehmer, im vorliegenden Fall also dazu, wie ihr Bild der Grammatik aussieht.

\section{Basiskonzeptualisierungen zur Grammatik}

Gemäß dem gerade geschilderten Vorgehen konnten acht Basiskonzeptualisierungen zur Grammatik aus den korpuslinguistischen Kollokationsdaten herausdestilliert werden. Sie werden im Folgenden kurz vorgestellt. Die Darstellung setzt jeweils bei den DWDS-Wortprofilen an und wird von Fall zu Fall durch Ergebnisse vertieft, die aus der DeReKo-Recherche stammen. Aus Raumgründen werde ich dabei nicht auf die statistischen Details eingehen können, die dafür jeweils als empirische Ausgangspunkte gedient haben. Das ist auch gar nicht nötig, weil hier ja nur die großen, typischen Linien von Interesse sind, weniger die vielen Details, die sich um vereinzelte Wörter (oder auch nur um einzelne Autoren) ranken können. Je geringer bestimmte Befunde statistisch hervorstechen, desto weniger sind sie schließlich für unsere Fragestellung von Bedeutung. Es sei daher auch daran erinnert, dass die korpuslinguistische Analyse hier lediglich eine dienende Funktion besitzt. Die korpuslinguistischen Ergebnisse stellen lediglich Durchgangsstadien dar, um durch sie mit abstrahierender Interpretation zum nicht-fachwissenschaftlich geprägten Wissen über 
die Grammatik vorzustoßen. Zur angemessenen Einschätzung der folgenden Aufzählung sei zudem festgehalten, dass die Reihenfolge bzw. Durchnummerierung der einzelnen Basiskonzeptualisierungen nur der (leichteren) Referenzierung dient. Dahinter stecken keine statistischen Gehalte oder (abstufenden) Wichtigkeitserwägungen.

BK 1: Grammatik ist gebunden an eine Einzelsprache, v. a. Deutsch, Latein, Englisch, Französisch, Griechisch.

BK 1 resultiert aus dem Umstand, dass Grammatik oft mit adjektivischen Attributen wie deutsch, lateinisch, englisch, französisch oder griechisch auftaucht (DeReKo auch hebräisch, Sanskrit). Die Grammatik wird demnach ganz überwiegend in einzelsprachlichen Ausprägungen konzeptualisiert. Dabei stehen die großen europäischen Sprachen deutlich im Vordergrund. Im Alltagsprachbewusstsein scheint Grammatik also kaum in universal(sprachlich)en Perspektiven wahrgenommen $\mathrm{zu}$ werden.

BK 2: Grammatik ist metaphorisch übertragbar auf nicht-sprachliche Entitäten. Für BK2 spricht die Tatsache, dass Grammatik recht häufig mit folgenden Genitiv-Attributen genutzt wird: Grammatik der Schöpfung, - des Gefühls, - des Lebens, - der Welt, - des Films, - der Musik. Semantisch sind diese Gebräuche recht heterogen. Sie gewinnen ihre Einheit lediglich daraus, dass für bestimmte nicht-sprachliche Seinsbereiche eine Regularität nach Art der Sprache angenommen wird. Das spricht allerdings dafür, dass der Grammatik prototypisch eine sehr hohe Regelhaftigkeit attestiert wird. Sie wird offensichtlich nicht selten durch einschlägige Attribut-Konstruktionen auf andere Bereiche übertragen. Womöglich könnte man daraus sogar schließen, dass die Grammatik kognitiv prototypisch für Regularität und Ordnung steht. Analog, allerdings mit anderer Akzentuierung, funktionieren wohl Formulierungen mit Mathematik bzw. Logik (vgl. etwa Mathematik/Logik der Schöpfung, - des Gefühls usw.). Einfach gesagt, Grammatik ist die Parade-Instanz für eine Ordnung, die man vielleicht nicht auf den ersten Blick erfasst, die aber durch bewusste Lernprozesse sichtbar werden kann.

BK 3: Grammatik existiert neben anderen Bereichen einer Sprache.

BK 3 ergibt sich daraus, dass Grammatik oft in syntaktischer Koordination gemeinsam mit Wörtern wie Rechtschreibung/Orthographie, Syntax, Vokabel und Wortschatz/Wörterbuch genutzt wird. Daraus wird ersichtlich, in welchen Komponenten Sprache geteilt und begriffen wird. Die Grammatik erscheint demnach als eine Sprachdimension, die in etwa mit den anderen Komponenten gleichge- 
ordnet ist. Einfach gesagt: Grammatik ist ein Teil der Sprache, nicht die Sprache. Ergänzt wird dieses Bild durch kookkurrente Substantive wie Logik, Trivium, Dialektik, Geometrie, Astronomie (DeReKo). Damit wird Grammatik als eine wissenschaftliche Grundlagendisziplin mit langer Tradition konzeptualisiert.

BK 4: Grammatik ist in einem Buch („einer Grammatik“) verzeichnet.

BK 4 lässt sich, anders als BK 1-3, nur etwas vermittelter aus den Korpusdaten ableiten. Bei den typischen Adjektivattributen tauchen nämlich neu und erst auf. Schaut man sich diese Belege näher an, wird klar, dass es dabei stets um die Grammatik als Buch geht. Bei den Verben, die Objekte mit Grammatik regieren, stehen auf derselben inhaltlichen Linie die Verben verfassen und entwerfen. Auch eine typische Konstruktion, in der Grammatik innerhalb einer Präpositionalphrase vorkommt, lautet Buch über Grammatik. Grammatik wird im Laienbewusstsein also oft mit explizit kodifizierten Regelwerken gleichgesetzt.

BK 5: Grammatik kann korrekt (richtig) bzw. inkorrekt/unkorrekt (falsch) sein.

Für BK 5 sprechen viele Befunde: Zunächst ist korrekt eins der typischen Adjektivattribute zu Grammatik, ferner sind Fehler und Schnitzer sehr typische Beiwörter zu grammatisch/grammatikalisch. Zahlreiche signifikante Adverbiale zu grammatisch/grammatikalisch weisen in dieselbe Richtung, so in erster Linie einwandfrei, falsch, fragwürdig, korrekt, richtig (DeReKo auch holprig, unschön, bedenklich, sauber, verunglückt, unausgereift, wohlgeformt, verquer als Kollokationen zu grammatisch/grammatikalisch). Aus den Daten wird folglich klar und deutlich ersichtlich, wie stark die Grammatik von Laien durch eine präskriptive Normbrille wahrgenommen und geprägt wird.

BK 6: Grammatik ist oft schwer und stellt ein Problem dar.

BK 6 steht offensichtlich mit BK 5 in Verbindung, soll hier jedoch als einzelne Basiskonzeptualisierung profiliert werden. Aus den Daten resultiert sie, weil Problem mit Grammatik eine typische Präpositionalphrase darstellt. Ferner ist schwer ein ganz typisches Adjektiv, zu dem Grammatik in eine Prädikativkonstruktion tritt (Grammatik ist schwer). Schwäche wiederum ist typisches Beiwort zum adjektivischen Attribut grammatikalisch. BK 6 verstärkt und intensiviert das Bild der Grammatik, was schon in BK 5 Gestalt angenommen hat.

BK 7: Grammatik ist Gegenstand bewusster Lernprozesse.

BK 7 ergänzt das laienlinguistische Bild und Wissen zur Grammatik um eine weitere wichtige Komponente. Sie wurzelt in verschiedenen Korpusbefunden. Dafür stehen typische Präpositionalphrasen (Kenntnis in -, interessieren für-, 
kümmern um -, beschäftigen mit Grammatik) sowie vor allem typische Satzkonstruktionen, in denen Grammatik als Objekt vorkommt: Demzufolge kann man Grammatik nämlich pauken, büffeln, beherrschen, lehren, lernen, üben, beibringen, studieren.

BK 8: Grammatik besteht aus einem Set von Regeln und stellt insofern eine als präskriptiv begriffene Regelungsinstanz dar.

Für BK 8 lassen sich hauptsächlich zahlreiche Kollokationen festmachen, die auf die typische Nutzung der Adjektive grammatisch/grammatikalisch zurückgehen. Sie stehen als Attribut nämlich überdurchschnittlich häufig vor den Substantiven Regel, Form, Struktur, Konstruktion. Dazu kann man weitere Befunde aus dem DeReKo stellen: Wenn Grammatik als Subjekt in einem AktivSatz auftaucht, ist vor allem mit den folgenden verbalen Fortsetzungen zu rechnen: bevorzugt, erlaubt, funktioniert, gebietet, gibt her, hält bereit, kennt, lässt $z u$, sagt, sieht vor, unterscheidet, verlangt, will, wünscht. Auch formal und Grammatik treten überdurchschnittlich häufig nebeneinander. Direkte inhaltliche Beziehungen der BK 8 gibt es mindestens zu BK 4 und 5, mittelbar auch zu BK 6 und 7.

Natürlich ließe sich lange darüber streiten, inwiefern aus den Kollokationsdaten in Teilen vielleicht andere Basiskonzeptualisierungen abstrahiert werden könnten. Auch deren Differenzierung und Klassifizierung wäre an einigen Stellen anders denkbar. Insgesamt ergibt sich nach dem oben Gesagten aber doch ein recht kohärentes Bild, das bei allen denkbaren Variationen im Kern ziemlich stabil bleiben dürfte. Demnach kann man das Bild, das Laien von der Grammatik haben, wie folgt zuspitzen und in Worte fassen:

Laien verstehen unter der Grammatik ein einzelsprachliches (BK 1) Inventar von Regeln und Strukturen (BK 8). Sie macht neben anderen Komponenten eine Sprache aus (BK 3) und ist in einem Buch aufgelistet (BK 4). Die zu befolgenden grammatischen Regeln und Strukturen sind Gegenstände bewusster Lernprozesse (BK 7). Sie sind oft schwierig und problematisch (BK 6) und führen daher immer wieder zu sprachlichen Fehlern, wenn sie nicht beachtet werden (BK 5). Die Grammatik gibt insofern ein einschlägiges Muster für Ordnungsstrukturen ab, das auch auf nicht-sprachliche Dinge übertragen werden kann (BK 2).

Wohlgemerkt: Mit der obigen Zusammenfassung soll nicht behauptet werden, dass alle Laien genau über dieses Bewusstsein der Grammatik verfügen. Es wird lediglich festgestellt, dass sich diese acht Haltungen aus dem öffentlichen, 
nicht-fachlichen Sprachgebrauch herausschälen lassen. Es kann durchaus sein, dass (einzelne, wenige, viele, sehr viele, alle) Laienlinguisten von den genannten acht Basiskonzeptualisierungen ausgehen und ihr sprachliches Verhalten darauf aufbauen. Bei anderen Laienlinguisten könnten dagegen nur einzelne Basiskonzeptualisierungen vorliegen, möglicherweise in typischen inhaltlichen Clustern (insgesamt dazu auch Hoffmeister 2020). Im Rahmen dieser Untersuchung konnte und sollte für solche Verteilungen aber kein direkter Nachweis erbracht werden. Wer an diesem Punkt auf Nummer sicher gehen möchte, müsste einen anderen methodologischen Ansatz wählen als den, der für die vorliegende Untersuchung gewählt wurde. Dann wäre auch in Rechenschaft zu ziehen, wie die nicht unerhebliche Spanne zwischen Laien und Experten (siehe oben Abschnitt 2) einigermaßen methodologisch kontrolliert in eine empirische Erhebung einfließen könnte. Es ist ja durchaus denkbar, dass sich einige Basiskonzeptualisierungen eher am Experten-Ende der Skala, andere eher am LaienEnde nachweisen lassen.

\section{Interpretation: Laiensicht vs. Expertensicht}

Die obige Identifikation von acht laienlinguistischen Basiskonzeptualisierungen zur Grammatik mag in toto inhaltlich wenig überraschen. Sicher sind einzelne Versatzstücke davon schon gut bekannt. Auf sie wurde in der Literatur immer wieder angespielt, mal ausführlich und explizit, mal eher implizit und beiläufig (vgl. z. B. Habermann 2010; von Polenz 1980; jüngst Beuge 2019). Dazu gehört beispielsweise die große laienlinguistische Neigung zu einer präskriptiven und fehlerfixierten Sichtweise auf Sprache, hier eben auf die Grammatik. In dieser Richtung habe ich beispielsweise schon davon gesprochen, dass im sprachlichen Alltag so etwas wie eine Richtig-oder-Falsch-Ideologie existiert (vgl. Klein 2018: 25-38), die stark mit einer kodex-zentrierten Sicht auf sprachliche Regeln verwoben ist (vgl. Klein 2014) ${ }^{1}$.

Mit der kleinschrittigen Ausfächerung von verschiedenen Basiskonzeptualisierungen lässt sich nun allerdings genauer festmachen, worin die Unterschiede zwischen einer Laien- und einer Expertensicht auf Grammatik bestehen und wie die Brisanz dieser Spannung zwischen Laien- und Expertenbegriffen im einzelnen beschaffen ist. Denn es ist ja nicht so, dass die acht Basiskonzeptuali-

1 Zur daraus resultierenden Kodex-Forschung siehe auch das Würzburger ZweiDat-Projekt unter http://kallimachos.de/zweidat/ (letzter Zugriff 03.06.2020). 
sierungen völlig sprachwissenschaftsferne Ansichten darstellen. Es gibt Gemeinsamkeiten und Differenzen zwischen den unterschiedlichen Konzeptualisierungen. Sie lassen sich wie folgt beschreiben:

Bei den BK 1, 2 und 3 kann man schnell eine weitgehende Übereinstimmung zwischen Experten- und Laiensicht konstatieren. Hier existiert derselbe Wissensbestand: Grammatik ist als eine Verkörperungsform von Sprache in der Regel an ein einzelnes Idiom gebunden. Das damit einhergehende Ordnungsdenken ist metaphorisch auf andere Sektoren übertragbar. Ziemlich weit liegen die Perspektiven dagegen bei BK 4 und 7 auseinander. In der Expertensicht ist die Grammatik keineswegs durch die Präsenz in einem Buch festgelegt ( $\neq$ BK 4). Jede natürliche Sprache besitzt in der Expertensicht eine Grammatik, auch wenn sie bisher noch nicht in einem Buch festgehalten wurde. Zudem ist das bewusste Lernen für Experten keine wesentliche Existenzbedingung für die Grammatik ( $\neq$ BK 7). Eher im Gegenteil: Der Erwerb der Grammatik ist in der Expertensicht etwas, was vor allem automatisch-spontan und wenig bewusst während des Erstspracherwerbs von statten geht. Manchen Sprachwissenschaftlern gilt die Grammatik sogar als angeboren, was geradezu das direkte Gegenteil eines bewussten Lernprozesses darstellt. Das absichtliche Erlernen der Grammatik erfolgt in diesen Perspektiven höchstens in einem späteren Prozess, der mit dem (schulischen) Schriftspracherwerb zusammenhängt.

Aus all dem folgt, dass auch bei BK 5, 6 und 8 unterschiedliche Horizonte dominieren, weil die Grammatik bei Laien und Experten in Teilen eben grundsätzlich anders verstanden wird. Auf den ersten Blick ist man bei BK 5 und 8 zwar noch relativ nah beieinander. Experten und Laien werden sich schnell darauf einigen, dass in der Grammatik Regeln existieren (= BK 8), die man auch brechen kann (= BK 5). Allerdings werden diese Regeln (bzw. Regelbrüche) von den Laien anders verstanden als von den Experten. Für Laien sind die grammatischen Regeln Wissensbestandteile, die in einem bewussten, schwierigen Lernprozess erworben werden. Für Experten ist klar, dass man solche Regeln spontan-naturwüchsig erwirbt, also eher mühelos und ohne großen Zwang. Für Laien stehen die grammatischen Regeln in einem Buch, das auch für die Durchsetzung und Überlieferung grammatischer Regeln sorgt. Für Experten sind die Regeln dagegen Instanzen, die sich aus dem realen Sprachgebrauch (und ggf. mentalen Repräsentationen) ergeben und dort auch tradiert werden. Regelbrüche sind insofern für Laien klare Zeichen für defizitäre, unabgeschlossene Lernprozesse. Für Experten könnte man - vielleicht etwas zugespitzt - feststellen, dass es in bestimmter Hinsicht eigentlich gar keine grammatischen Regelbrüche geben kann, wenn man einmal von mündlichen Versprechern und schriftlichen Vertippern absieht. Angebliche Regelbrüche können sich demnach nämlich 
dadurch erklären lassen, dass man nur die eine (gebrochene) Regel durch eine andere (befolgte) Regel ersetzt. In dieser Sicht wird jeder Sprachgebrauch regulär.

Man erkennt: An einem wesentlichen Punkt reden Experten und Laien potentiell von ziemlich unterschiedlichen Dingen, wenn sie von der Grammatik und ihren Regeln sprechen. Man könnte auch sagen, dass die Grammatik und ihre Regeln bei Experten und Laien einen ganz unterschiedlichen ontologischen Status besitzen.

Das Ganze lässt sich weiterdenken. Angesichts dieser Klärung wird es nun nämlich etwas verständlicher, warum das Verhältnis zwischen Experten und Laien auf dem Feld der Sprache - wie eingangs ausgeführt - von tiefen Konflikten durchzogen ist. Sie wurzeln - zumindest zum Teil und womöglich vor allem auf dem Gebiet der Grammatik - darin, dass man nicht dieselben Dinge versteht, wenn man dieselben Wörter - nämlich Grammatik und grammatisch/grammatikalisch - nutzt. Auf diesem Feld existiert offensichtlich ein großes Missverständnispotenzial. Es lässt sich dadurch rekonstruieren, dass man die Kollokationen des öffentlichen Sprachgebrauchs ernst nimmt, daraus Basiskonzeptualisierungen ableitet und mit den fachlichen Gegebenheiten der Sprachwissenschaft vergleicht. Vermutlich wird man auch die großen Unterschiede, die hinsichtlich des Status sprachlicher Bewertungen zwischen Laien und Experten bestehen, auf solche konzeptuellen Differenzen zurückführen können. Für Laien ist die Bewertung von Sprache immer schon tief in ihrem grundlegenden Grammatik-Begriff verankert. Aus dem Grammatik-Begriff der Experten ergibt sich ein solcher Zusammenhang gerade nicht.

\section{Fazit}

Die Frage, was linguistische Laien über die (deutsche) Grammatik denken, lässt sich zweifellos mit unterschiedlichen Methoden beantworten. Hier wurde ein Weg vorgeschlagen, der sich am öffentlichen Sprachgebrauch und der Nutzung der Wörter Grammatik und grammatisch/grammatikalisch orientiert. Durch die Nutzung einschlägiger Kollokationen konnten so acht laienlinguistische Basiskonzeptualisierungen ermittelt werden. Sie zeigen ein differenziertes Verständnis. Es stimmt an einigen Punkten mit der Expertensicht überein. An anderen Stellen ergeben sich freilich auch fundamentale Unterschiede. Mit ihnen kann man erklären, warum das Verhältnis von Laienlinguistik und Fachwissenschaft spannungsvoll ist. Wer daran interessiert ist, dieses prekäre Verhältnis ein Stück weit zu entspannen, sollte also auf dem Feld der Grammatik insbesondere 
die laienlinguistischen Basiskonzeptualisierungen 4, 5, 6 und 8 in Augenschein nehmen und ihre Konsequenzen für eine Sicht auf Sprache beachten. Sie zeugen von den großen Unterschieden zwischen Laien und Experten bei der Thematisierung von Grammatik. Daraus können große Missverständnisse resultieren.

Möglicherweise steckt darin auch ein Baustein zu einer Erklärung, warum die öffentlichen Sprachdiskussionen und die sprachwissenschaftlichen Forschungsperspektiven seit dem 19. Jahrhundert nur noch selten wirklich in dieselbe Richtung laufen. In dieser Sicht könnte der vorliegende Diskussionsbeitrag vielleicht dazu anregen, wissenschaftliche und Alltagsperspektiven auf Sprache (und Grammatik) stärker zu vermitteln und das Gespräch zwischen Sprachwissenschaftlern und Laien etwas fruchtbarer zu machen als oft üblich. Das ist natürlich eine gewollt optimistische Sicht auf die Dinge. In Anbetracht der starken Dissonanzen zwischen Sprachwissenschaft und Sprachöffentlichkeit muss man freilich auch damit rechnen, dass der Graben zwischen den beiden Instanzen viel zu tief sein könnte, als dass er durch eine simple Behebung von wortsemantischen Missverständnissen überbrückt werden könnte.

\section{Literaturverzeichnis}

Adler, Astrid \& Albrecht Plewnia (2019): Die Macht der großen Zahlen. Aktuelle Spracheinstellungen in Deutschland. In Ludwig M. Eichinger \& Albrecht Plewnia (Hrsg.), Neues vom heutigen Deutsch. Empirisch - methodisch - theoretisch, 141-162. Berlin, Boston: De Gruyter.

Anders, Christina Ada (2010): Die wahrnehmungsdialektologische Rekodierung von laienlinguistischem Alltagswissen. In Christina Ada Anders, Markus Hundt \& Alexander Lasch (Hrsg.), Perceptual dialectology. Neue Wege der Dialektologie, 67-87. Berlin, New York: De Gruyter.

Anders, Christina Ada, Markus Hundt \& Alexander Lasch (Hrsg.) (2010): Perceptual dialectology. Neue Wege der Dialektologie. Berlin, New York: De Gruyter.

Andresen, Carl-Gustav (1876): Ueber deutsche Volksetymologie. Heilbronn: Henninger.

Andresen, Helga (1985): Schriftspracherwerb und die Entstehung von Sprachbewußtheit. Opladen: Westdeutscher Verlag.

Antos, Gerd (1996): Laien-Linguistik. Studien zu Sprach- und Kommunikationsproblemen im Alltag. Am Beispiel von Sprachratgebern und Kommunikationstrainings. Tübingen: Niemeyer.

Berthele, Raphael (2010): Der Laienblick auf sprachliche Varietäten: Metalinguistische Vorstellungswelten in den Köpfen der Deutschschweizerinnen und Deutschschweizer. In Christina Ada Anders, Markus Hundt \& Alexander Lasch (Hrsg.), Perceptual dialectology. Neue Wege der Dialektologie, 245-267. Berlin, New York: De Gruyter. 
Beuge, Patrick (2014): Was ist gutes Deutsch aus Sicht linguistischer Laien? Zeitschrift für Dialektologie und Linguistik LXXXI (2), 129-150.

Beuge, Patrick (2019): Was ist gutes Deutsch? Eine qualitative Analyse laienlinguistischen Sprachnormwissens. Berlin, Boston: De Gruyter.

Borst, Arno (1957-1963): Der Turmbau von Babel. Geschichte der Meinungen über Ursprung und Vielfalt der Sprachen und Völker. Stuttgart: Hierseman.

Christen, Helen (2010): Was Dialektbezeichnungen und Dialektattribuierungen über alltagsweltliche Konzeptualisierungen sprachlicher Heterogenität verraten. In Christina Ada Anders, Markus Hundt \& Alexander Lasch (Hrsg.), Perceptual dialectology. Neue Wege der Dialektologie, 269-290. Berlin, New York: De Gruyter.

Cuonz, Christina (2014): Sprachliche Werturteile von Laien. Eine sozio-kognitive Analyse. Tübingen: Francke.

Davies, Winifred V. (2010): Die Rolle (laien-) linguistischer Mythen bei der Reproduktion (sozio-) linguistischer Normen. In Christina Ada Anders, Markus Hundt \& Alexander Lasch (Hrsg.), Perceptual dialectology. Neue Wege der Dialektologie, 385-408. Berlin, New York: De Gruyter.

Deutsche Akademie für Sprache und Dichtung/Union der Deutschen Akademien der Wissenschaften (Hrsg.) (2013): Reichtum und Armut der deutschen Sprache. Erster Bericht zur Lage der deutschen Sprache. Berlin, Boston: De Gruyter.

Deutsche Akademie für Sprache und Dichtung/Union der Deutschen Akademien der Wissenschaften (Hrsg.) (2017): Vielfalt und Einheit der deutschen Sprache. Zweiter Bericht zur Lage der deutschen Sprache. Tübingen: Stauffenburg.

Eichinger, Ludwig M. et al. (2009): Aktuelle Spracheinstellungen in Deutschland. Erste Ergebnisse einer bundesweiten Repräsentativumfrage. Mannheim: Institut für deutsche Sprache.

Ernst, Oliver, Jan Claas Freienstein \& Lina Schaipp (2011): Populäre Irrtümer über Sprache. Stuttgart: Reclam.

Fögen, Thorsten (2000): Patrii sermonis egestas. Einstellungen lateinischer Autoren zu ihrer Muttersprache. Ein Beitrag zum Sprachbewußtsein in der römischen Antike. München, Leipzig: Saur.

Gärtig, Anne-Kathrin, Albrecht Plewnia \& Astrid Rothe (2010): Wie Menschen in Deutschland über Sprache denken. Ergebnisse einer bundesweiten Repräsentativerhebung zu aktuellen Spracheinstellungen. Mannheim: Institut für deutsche Sprache.

Glück, Helmut (1979): Die preußisch-polnische Sprachenpolitik. Eine Studie zur Theorie und Methodologie der Forschung über Sprachenpolitik, Sprachbewußtsein und Sozialgeschichte am Beispiel der preußisch-deutschen Politik gegenüber der polnischen Minderheit vor 1914. Hamburg: Buske.

Habermann, Mechthild (Hrsg.) (2010): Grammatik wozu? Vom Nutzen des Grammatikwissens in Alltag und Schule. Mannheim, Zürich: Duden-Verlag.

Hennig, Mathilde (2016): Über welche grammatischen Konzepte verfügen wir? Ein empirischer Beitrag zur Grammatikbenutzungsforschung und Transferwissenschaft. Deutsche Sprache 44, 1-22.

Hoffmeister, Toke (2019): Laien als Experten und Experten als Laien. Zur Problematik eines etablierten Begriffspaares. Linguistik online 99, 6/19, 151-174.

Hoffmeister, Toke (2020): Subjektive Grammatikalitätstheorien. Entstehung, Verbreitung und forschungspraktische Konsequenzen. Deutsche Sprache 3/2020, 233-248. 
Huber, Wolfgang (1984): Kulturpatriotismus und Sprachbewusstsein. Studien zur deutschen Philologie des 17. Jahrhunderts. Frankfurt a. M. u. a.: Lang.

Hundt, Markus (1992): Einstellungen gegenüber dialektal gefärbter Standardsprache. Eine empirische Untersuchung zum Bairischen, Hamburgischen, Pfälzischen und Schwäbischen. Stuttgart: Steiner.

Hundt, Markus (2010): Bericht über die Pilotstudie „Laienlinguistische Konzeptionen deutscher Dialekte“. In Christina Ada Anders, Markus Hundt \& Alexander Lasch (Hrsg.), Perceptual dialectology. Neue Wege der Dialektologie, 179-219. Berlin, New York: De Gruyter.

Hundt, Markus (2018): Wahrnehmungsdialektologie - quo vadis? In Alexandra N. Lenz \& Albrecht Plewnia (Hrsg.): Variation - Norm(en) - Identität(en), 99-126. Berlin, Boston: De Gruyter.

Klein, Wolf Peter (1992): Am Anfang war das Wort. Theorie- und wissenschaftsgeschichtliche Elemente frühneuzeitlichen Sprachbewußtseins. Berlin: Akademie-Verlag.

Klein, Wolf Peter (2004): Deskriptive statt präskriptiver Sprachwissenschaft!? Über ein sprachtheoretisches Bekenntnis und seine analytische Präzisierung. Zeitschrift für germanistische Linguistik 32 (3), 376-405.

Klein, Wolf Peter (2014): Gibt es einen Kodex für die Grammatik des Neuhochdeutschen und, wenn ja, wie viele? Oder: Ein Plädoyer für Sprachkodexforschung. In Albrecht Plewnia \& Andreas Witt (Hrsg.), Sprachverfall? Dynamik - Wandel - Variation, 219-242. Berlin, Boston: De Gruyter.

Klein, Wolf Peter (2018): Sprachliche Zweifelsfälle im Deutschen. Theorie, Praxis, Geschichte. Berlin: De Gruyter.

Klotzsche, Felix \& Philip Reich (2016): Einstellungen zu Variationen in der Orthografie. Eine Umfrage zu Einflüssen von Orthografievariation. In Paul Rössler (Hrsg.), Standardisierungsprozesse und Variation. Beiträge zur Engführung von Standardsprachenforschung und Variationslinguistik, 21-54. Frankfurt a. M.: Lang.

Leweling, Beate (2005): Reichtum, Reinigkeit und Glanz. Sprachkritische Konzeptionen in der Sprachreflexion des 18. Jahrhunderts; ein Beitrag zur Sprachbewusstseinsgeschichte. Frankfurt a. M.: Lang.

Meciarova, Martina (2015): Semantische Remotivierung als Produkt laienhafter Reflexion über Sprache. Begründungen von Wortwahlen als Form sprachlicher Verstärkung. Hamburg: Buske.

Müller, Roman (2001): Sprachbewußtsein und Sprachvariation im lateinischen Schrifttum der Antike. München: Beck.

Niederhauser, Jürg (1997): Populärwissenschaftliches Schreiben über sprachliche Fragen und linguistische Themen. In Kirsten Adamzik, Gerd Antos \& Eva-Maria Jakobs (Hrsg.), Domänen- und kulturspezifisches Schreiben, 203-221. Frankfurt a. M. u. a.: Lang.

Niedzielski, Nancy A. \& Dennis R. Preston (2003): Folk linguistics. Berlin, New York: Mouton de Gruyter.

Olschansky, Heike (1996): Volksetymologie. Berlin, Boston: De Gruyter.

Peter, Klaus (2011): Textbewertungen. Eine empirische Untersuchung zu Sprachbewusstheit und Spracheinstellungen. Tübingen: Stauffenburg.

Perkuhn, Rainer \& Cyril Belica (2004): Eine kurze Einführung in die Kookkurrenzanalyse und syntagmatische Muster. Institut für Deutsche Sprache, Mannheim. (http://www1.idsmannheim.de/kl/misc/tutorial.html, letzter Zugriff 03.06.2020).

Plewnia, Albrecht \& Astrid Rothe (2012): Sprache - Einstellungen - Regionalität. In Ludwig Eichinger, Albrecht Plewnia, Christiane Schöl \& Gerhard Stickel (Hrsg.), Sprache und Ein- 
stellungen. Spracheinstellungen aus sprachwissenschaftlicher und sozialpsychologischer Perspektive, 9-118. Tübingen: Narr.

Polenz, Peter von (1980): Wie man über Sprache spricht. Über das Verhältnis zwischen wissenschaftlicher und natürlicher Beschreibungssprache in Sprachwissenschaft und Sprachlehre. Mannheim: Duden-Verlag.

Regener, Irena (2000): Selbstidentifikation via Varietätengebrauch. Sprachverhalten und Spracheinstellungen in der Berliner Sprachgemeinschaft der 90er Jahre. Linguistik online 7 (3).

Scharloth, Joachim (2005): Sprachnormen und Mentalitäten. Sprachbewusstseinsgeschichte in Deutschland im Zeitraum von 1766 bis 1785 . Tübingen: Niemeyer.

Schmidlin, Regula, Heike Behrens \& Hans Bickel (Hrsg.) (2015): Sprachgebrauch und Sprachbewusstsein. Implikationen für die Sprachtheorie. Berlin, Boston: De Gruyter.

Spiekermann, Helmut (2010): Visualisierungen von Dialekten: Ein Beitrag zum Nutzen der Laiendialektologie. In Christina Ada Anders, Markus Hundt \& Alexander Lasch (Hrsg.), Perceptual dialectology. Neue Wege der Dialektologie, 221-244. Berlin, New York: De Gruyter.

Strauss, Lina (2018): Rhetorikratgeber als Beispiel für Laienlinguistik. Eine Diskursanalyse. Berlin: Metzler.

Wilton, Antje \& Martin Stegu (Hrsg.) (2011): Applied folk linguistics. Amsterdam u. a: Benjamins.

Ziegler, Evelyn (1999): Deutsch im 19. Jahrhundert. Normierungsprinzipien und Spracheinstellungen. In Helga Bister-Broosen (Hrsg.), Beiträge zur historischen Stadtsprachenforschung, 79-101. Wien: Ed. Praesens. 